\title{
MHD Free Convection Flow past an Inclined Stretching Sheet with Considering Viscous Dissipation and Radiation
}

\author{
Maruf Hasan1, Enamul Karim1, Abdus Samad² \\ ${ }^{1}$ Department of Mathematics, Comilla University, Comilla, Bangladesh \\ ${ }^{2}$ Department of Applied Mathematics, University of Dhaka, Dhaka, Bangladesh \\ Email:marufek@yahoo.com
}

How to cite this paper: Hasan, M., Karim, E. and Samad, A. (2017) MHD Free Convection Flow past an Inclined Stretching Sheet with Considering Viscous Dissipation and Radiation. Open Journal of Fluid Dynamics, 7, 152-168.

https://doi.org/10.4236/ojfd.2017.72010

Received: April 5, 2017

Accepted: May 8, 2017

Published: May 11, 2017

Copyright $\odot 2017$ by authors and Scientific Research Publishing Inc. This work is licensed under the Creative Commons Attribution International License (CC BY 4.0).

http://creativecommons.org/licenses/by/4.0/

\begin{abstract}
The present study concentrates on the analysis of MHD free convection flow past an inclined stretching sheet. The viscous dissipation and radiation effects are assumed in the heat equation. Approximation solutions have been derived for velocity, temperature, concentration, Nusselt number, skin friction and Sherwood number using Nachtsheim-Swigert shooting iteration technique along with the six-order Runge-Kutta iteration scheme. Graphs are plotted to find out the characteristics of different physical parameters. The variations of physical parameters on skin friction coefficient, Nusselt number and Sherwood number are displayed via table.
\end{abstract}

\section{Keywords}

Viscous Dissipation, Magnetic Field, Skin Friction, Eckert Number

\section{Introduction}

In recent years, considerable interest has been shown in investigating radiation interaction with natural convection flow commonly known as free convection for heat transfer in fluid. This is due to the significant role of thermal radiation in the surface heat transfer when convection heat transfer is small particularly in free convection problems. Again the boundary layer flow on continuous surfaces is an important type of flow which occurs in a number of technical processes. Examples are paper production, crystal growing and glass blowing, aerodynamics extrusion of plastic sheets and fibers. Thus, the study of heat transfer has become important industrially for determining the quality of the final product. Laminar natural convection flow and heat transfer of fluid with and without heat source in channels with constant wall temperature have been extensively studied 
by Ostrach [1]. Hossain and Takhar [2] analyzed the effect of radiation effects on free convection flow of a gas past a semi infinite flat plate using the Cogley-Vincnitine-Giles equilibrium model. Ali et al. [3] studied the radiation effect on natural convection flow over a vertical surface in a gray gas. Following Ali et al., Mansour [4] studied the interaction of mixed convection with thermal radiation in laminar boundary layer flow over a horizontal, continuous moving sheet with suction/injection. Alabraba et al. [5] studied the same problem considering magnetic effect taking into account the chemical reaction and soret-doufour effects. Sattar and Kalim [6] made a study of the combined unsteady free convection dynamic boundary layer and thermal radiation boundary layer on a semi-infinite vertical plate by using the Rosseland diffusion approximation. Chen [7] investigated natural convection flow over a permeable surface with variable wall temperature and concentration.

The viscous dissipation effect plays an important role in natural convection. Natural convection flow is often encountered in the cooling of nuclear reactors. Viscous dissipation effects on non-linear MHD flow in a porous medium over a stretching porous surface have been studied by S.P. Anjali Devi and B. Ganga [8]. Jha and Ajibade [9] studied the effect of viscous dissipation on natural convection flow between vertical parallel plates with time-periodic boundary conditions. Ferdows et al. [10] described that in the presence of uniform magnetic field with viscous dissipation at the wall, the thermophoretic parameter is one of the most useful parameters to control the boundary layer of the fluid.

The analysis and discussion of natural convection flow, the viscous dissipation effect is generally ignored but here considered the combined effect of viscous dissipation and radiation on free convection flow an inclined stretching sheet.

\section{Mathematical Analysis}

A steady-state two-dimensional heat and mass transfer flow of an electrically conducting viscous incompressible fluid along an isothermal stretching permeable inclined sheet with an angle $\alpha$ to the vertical embedded in a porous medium with heat generation/absorption is considered. A strong magnetic field is applied in the $y$-axis direction. Here the effect of the induced magnetic field is neglected in comparison to the applied magnetic field. The electrical current flowing in the fluid gives rise to an induced magnetic field if the fluid were an electrical insulator, but here we have taken the fluid to be electrically conducting. Hence, only the applied magnetic field of strength $B_{0}$ plays a role which gives rise to magnetic forces $F=\frac{\sigma B_{0}^{2} u}{\rho}$ in $x$-direction, $\sigma$ where is the electrical conductivity assumed to be directly proportional to the $x$-translational velocity $(u)$ of the fluid found by Helmy [11] and $\rho$ is the density of the fluid. Two equal and opposite forces are introduced along the $x$ - axis so that the sheet is stretched keeping the origin fixed as shown in Figure 1.

The fluid is considered to be gray, absorbing emitting radiation but non-scattering medium and the Rosseland approximation is used to describe the radia- 


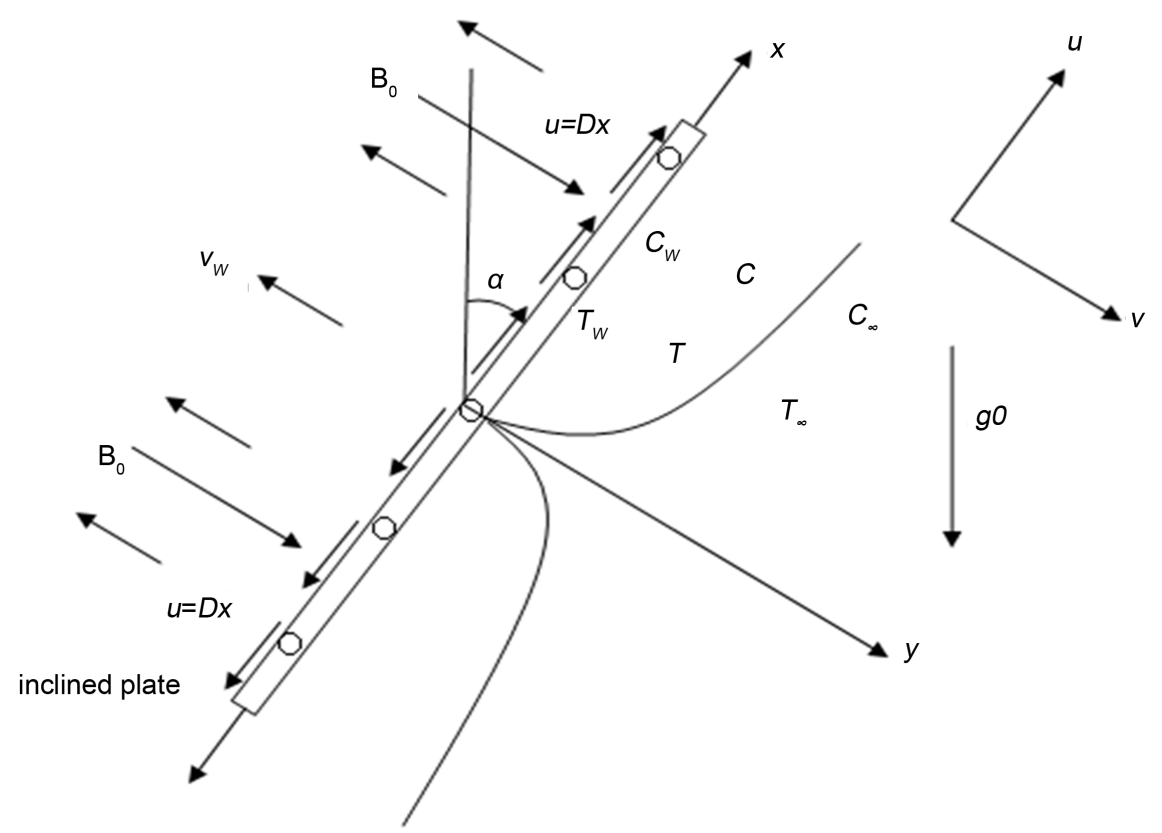

Figure 1. Physical model and coordinate system.

tion heat flux in the energy equation. The radiative heat flux in the $x$-direction is negligible to the flux in the $y$-direction. The plate temperature and concentration are initially raised to $T_{w}$ and $C_{w}$ respectively which are thereafter maintained constant. The ambient temperature of the flow is $T_{\infty}$ and the concentration of the uniform flow is $C_{\infty}$.

Under the usual boundary layer and Boussinesq approximations and using the Darcy-Forchhemier model, the flow and heat transfer in the presence of radiation are governed by the following equations.

Continuity equation

$$
\frac{\partial u}{\partial x}+\frac{\partial v}{\partial y}=0
$$

Momentum equation

$$
u \frac{\partial u}{\partial x}+v \frac{\partial u}{\partial y}=v \frac{\partial^{2} u}{\partial y^{2}}+g_{0} \beta\left(T-T_{\infty}\right) \cos \alpha-\frac{\sigma B_{0}^{2} u}{\rho}-\frac{v u}{k}-\frac{b u^{2}}{k}
$$

Energy equation

$$
u \frac{\partial T}{\partial x}+v \frac{\partial T}{\partial y}=\frac{\kappa}{\rho c_{p}} \frac{\partial^{2} T}{\partial y^{2}}+\frac{Q_{0}}{\rho c_{p}}\left(T-T_{\infty}\right)+\frac{v}{c_{p}}\left(\frac{\partial u}{\partial y}\right)^{2}-\frac{1}{\rho c_{p}} \frac{\partial q_{r}}{\partial y}
$$

Concentration equation

$$
u \frac{\partial C}{\partial x}+v \frac{\partial C}{\partial y}=D_{m} \frac{\partial^{2} C}{\partial y^{2}}
$$

where $u$ and $v$ are the velocity components in the $x$-direction and $y$-direction respectively, $v$ is the kinematic viscosity, $g_{0}$ is the acceleration due to gravity, $\beta$ is the volumetric coefficient of thermal expansion, $\alpha$ is the angle of inclination, $k$ is the Darcy permeability constant, $T$ and $T_{\infty}$ are the fluid tem- 
perature within the boundary layer and in the free-stream respectively, while $C$ is the concentration of the fluid within the boundary layer, $\sigma$ is the electric conductivity, $B_{0}$ is the uniform magnetic field strength (magnetic induction), $\rho$ is the density of the fluid, $\kappa$ is the thermal conductivity of the fluid, $c_{p}$ is the specific heat at constant pressure, $Q_{0}$ is the volumetric rate of heat generation/absorption and $D_{m}$ is the chemical molecular diffusivity.

The corresponding boundary conditions are

$$
\left.\begin{array}{l}
u=D x, v=v_{w}(x), T=T_{w}, C=C_{w} \text { at } y=0 \\
u=0, T=T_{\infty}, C=C_{\infty} \text { at } y \rightarrow \infty
\end{array}\right\}
$$

where $D(>0)$ is a constant, $v_{w}(x)$ is a velocity component at the wall having positive value to indicate suction, $T_{w}$ is the uniform sheet temperature and $C_{w}$ is the concentration of the fluid at the sheet.

By using Rosseland approximation, $q_{r}$ takes the form

$$
q_{r}=-\frac{4 \sigma_{1}}{3 \kappa_{1}} \frac{\partial T^{4}}{\partial y}
$$

where $\sigma_{1}$ is the Stefan-Boltzmann constant and $\kappa_{1}$ is the mean absorption coefficient. It is assumed that the temperature difference within the flow are sufficiently small such that $T^{4}$ may be expressed as a linear function of temperature.

$$
\text { Thus } T^{4} \cong 4 T_{\infty}^{3} T-3 T_{\infty}^{3}
$$

Using the Equations (6) and (7) in Equation (3), we get

$$
u \frac{\partial T}{\partial x}+v \frac{\partial T}{\partial y}=\frac{\kappa}{\rho c_{p}} \frac{\partial^{2} T}{\partial y^{2}}+\frac{Q_{0}}{\rho c_{p}}\left(T-T_{\infty}\right)+\frac{v}{c_{p}}\left(\frac{\partial u}{\partial y}\right)^{2}+\frac{16 \sigma_{1} T_{\infty}^{3}}{3 \rho c_{p} \kappa_{1}} \frac{\partial^{2} T}{\partial y^{2}}
$$

\subsection{Similarity Analysis}

In order to obtain similarity solution for the problem under consideration, we may take the following suitable similarity variables

$$
\eta=y \sqrt{\frac{D}{v}}, \psi=\sqrt{D v} x f(\eta), \theta(\eta)=\frac{T-T_{\infty}}{T_{w}-T_{\infty}}, \phi(\eta)=\frac{C-C_{\infty}}{C_{w}-C_{\infty}}
$$

where $\psi$ is the stream function, $\eta$ is the dimensionless distance normal to the sheet, $f$ is the dimensionless stream function, $\theta$ is the dimensionless fluid temperature and $\phi$ is the dimensionless concentration.

Since $u=\frac{\partial \psi}{\partial y}$ and $v=-\frac{\partial \psi}{\partial x}$ we have the velocity components from Equation (6) given by

$$
u=D x f^{\prime}(\eta), v=-\sqrt{D v} f(\eta)
$$

where prime denotes the derivative with respect to $\eta$.

Now introducing the similarity variables from Equation (9) and using Equation (10), Equations (2), (8) and (4) are reduced to the dimensionless equations given by 


$$
\begin{gathered}
f^{\prime \prime \prime}+f f^{\prime \prime}+\gamma \theta \cos \alpha-\left(M+\frac{1}{D a \cdot R e}\right) f^{\prime}-\left(1+\frac{F s}{D a}\right)\left(f^{\prime}\right)^{2}=0 \\
\theta^{\prime \prime}+\operatorname{Pnf} \theta^{\prime}+\operatorname{Pn} \theta Q+E c P n\left(f^{\prime \prime}\right)^{2}=0 \\
\phi^{\prime \prime}+\operatorname{Scf} \phi^{\prime}=0
\end{gathered}
$$

where $\gamma=\frac{G r_{x}}{R e_{x}^{2}}=\frac{g_{0} \beta\left(T_{w}-T_{\infty}\right)}{D^{2} x}$ is the buoyancy parameter, $M=\frac{\sigma B_{0}^{2}}{\rho D}$ is the magnetic field parameter, $D a=\frac{k}{x^{2}}$ is the local Darcy number, $R e_{x}=\frac{x^{2} D}{v}$ is the Reynolds number, $F s=\frac{b}{x}$ is the Forchhemier number, $\operatorname{Pr}=\frac{\mu c_{p}}{\kappa}$ is the Prandtl number, $Q=\frac{Q_{0}}{\rho c_{p} D}$ is the heat source $(Q>0) /$ sink $(Q<0)$ parameter, $E c=\frac{D^{2} x^{2}}{c_{p}\left(T_{w}-T_{\infty}\right)}$ is the Eckert number, $N=\frac{\kappa \kappa_{1}}{4 \sigma_{1} T_{\infty}^{3}}$ is the radiation parameter, $P n=\frac{3 N \cdot P r}{4+3 N}$ and $S c=\frac{v}{D_{m}}$ is the Schmidt number.

The transformed boundary conditions are

$$
f=F w, f^{\prime}=1, \theta=1, \phi=1 \text { at } \eta=0 f^{\prime}=1, \theta=1, \phi=1 \quad \text { as } \eta \rightarrow \infty
$$

where $F w=-\frac{v_{w}}{\sqrt{D v}}$ is the suction parameter for $F w>0$.

The nonlinear ordinary differential Equations (11), (12) and (13) under the boundary conditions (14) are solved numerically for various values of the parameters entering into the problems.

\subsection{Skin Friction, Rate of Heat and Mass Transfer}

The parameters of engineering interest for the present problem are the skin friction coefficient $\left(C_{f}\right)$, local Nusselt number $\left(N u_{x}\right)$ and Sherwood number (Sh) which indicate physically the wall shear stress, the rate of heat transfer and the local surface mass flux respectively. From the following definitions

$$
\tau_{w}=\mu\left(\frac{\partial u}{\partial y}\right)_{y=0}, q_{w}=-\kappa\left(\frac{\partial T}{\partial y}\right)_{y=0}, M_{w}=-D_{m}\left(\frac{\partial C}{\partial y}\right)_{y=0}
$$

where $\mu$ is the viscosity, $\kappa$ is the thermal conductivity and $D_{m}$ is the mass diffusivity. The dimensionless local wall shear stress, local surface heat flux and the local surface mass flux for an impulsively started plate are respectively obtained as

$$
\begin{gathered}
\frac{\sqrt{v} \tau_{w}}{x \mu D^{3 / 2}}=f^{\prime \prime}(0), C_{f}=\frac{2 \tau_{w}}{\rho U^{2}}=2\left(R e_{x}\right)^{-1 / 2} f^{\prime \prime}(0) \\
\frac{q_{w}}{\kappa\left(T_{w}-T_{\infty}\right)} \sqrt{\frac{v}{D}}=-\theta^{\prime}(0), N u_{x}=\frac{q_{w} x}{\kappa\left(T_{w}-T_{\infty}\right)}=-\left(R e_{x}\right)^{\frac{1}{2}} \theta^{\prime}(0) \\
\frac{M_{w}}{D_{m}\left(C_{w}-C_{\infty}\right)} \sqrt{\frac{v}{D}}=-\phi^{\prime}(0), S h=\frac{M_{w} x}{D_{m}\left(C_{w}-C_{\infty}\right)}=-\left(R e_{x}\right)^{\frac{1}{2}} \phi^{\prime}(0)
\end{gathered}
$$


where $R e_{x}=\frac{U x}{v}=\frac{x^{2} D}{v}$ is the Reynolds number. Hence the values proportional to the skin-friction coefficient, Nusselt number and Sherwood number are $f^{\prime \prime}(0),-\theta^{\prime}(0)$ and $-\phi^{\prime}(0)$ respectively.

\subsection{Numerical Computation}

The numerical solutions of the non-linear differential Equations (11)-(13) under the boundary conditions (14) have been performed by applying a shooting method namely Nachtsheim and Swigert [12] iteration technique (guessing the missing values) along with sixth order Runge-Kutta iteration scheme. We have chosen a step size $\Delta \eta=0.01$ to satisfy the convergence criterion of $10^{-6}$ in all cases. The value of $\eta_{\infty}$ has been found to each iteration loop by $\eta_{\infty}=\eta_{\infty}+\Delta \eta$. The maximum value of $\eta_{\infty}$ to each group of parameters

$\alpha, E c, F w, M, N, \operatorname{Pr}, Q, S c, \quad \gamma, D a$ and $F s$ has been determined when the values of the unknown boundary conditions at $\eta=0$ not change to successful loop with error less than $10^{-6}$.

In order to verify the effects of the step size $\Delta \eta$, we have run the code for our model with three different step sizes as $\Delta \eta=0.01, \Delta \eta=0.001$ and $\Delta \eta=0.005$ in each case we have found excellent agreement among them shown in Figures 2-4.

\section{Results and Discussion}

For the purpose of discussing the results of the flow field represented in the Figure 1, the numerical calculations are presented in the form of non-dimensional velocity, temperature and concentration profiles. The value of buoyancy parameter $\gamma$ is taken to be positive to represent cooling of the plate. The parame-

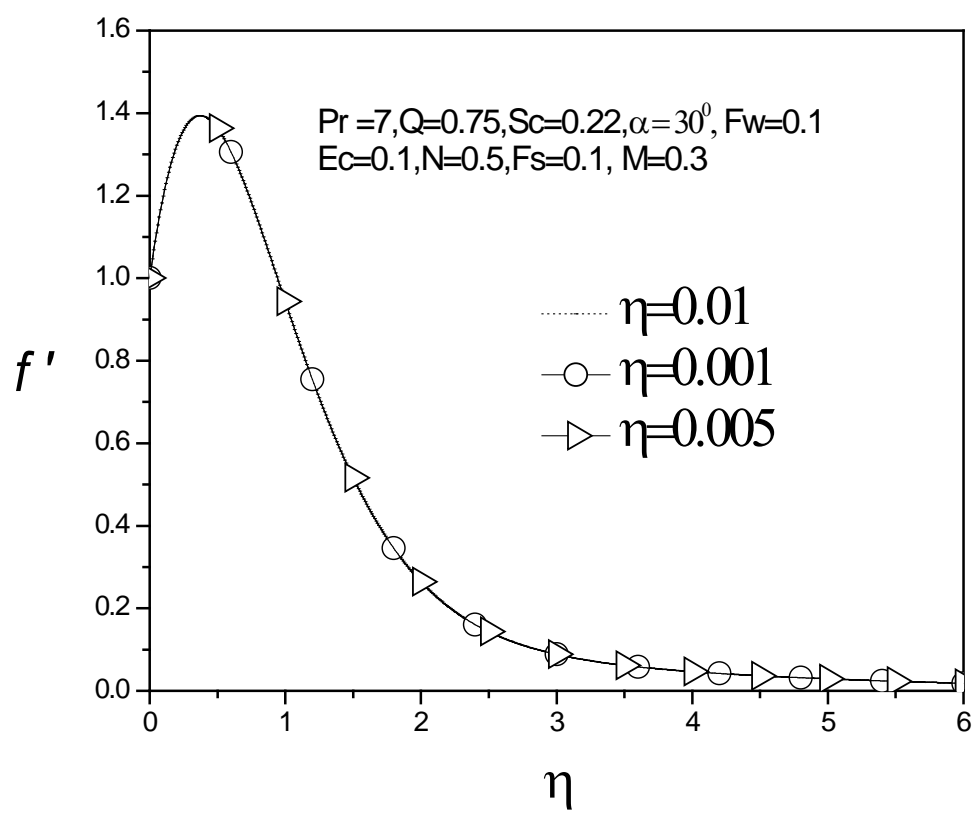

Figure 2. Velocity profiles for different step sizes. 


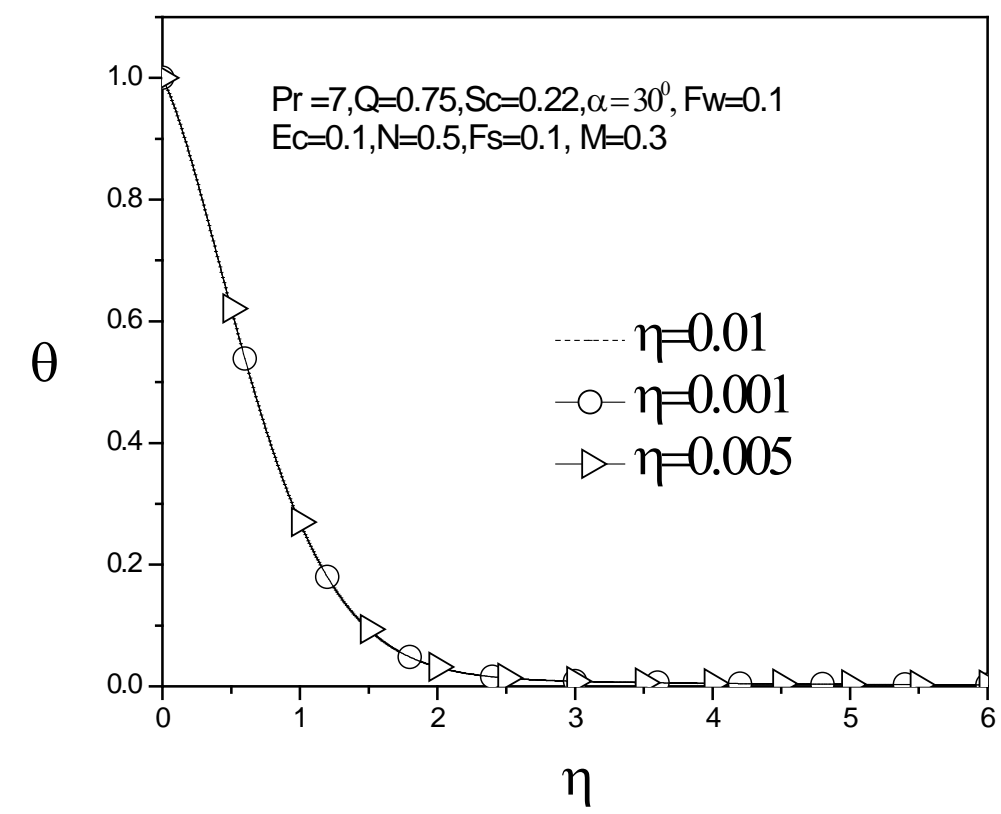

Figure 3. Temperature profiles for different step sizes.

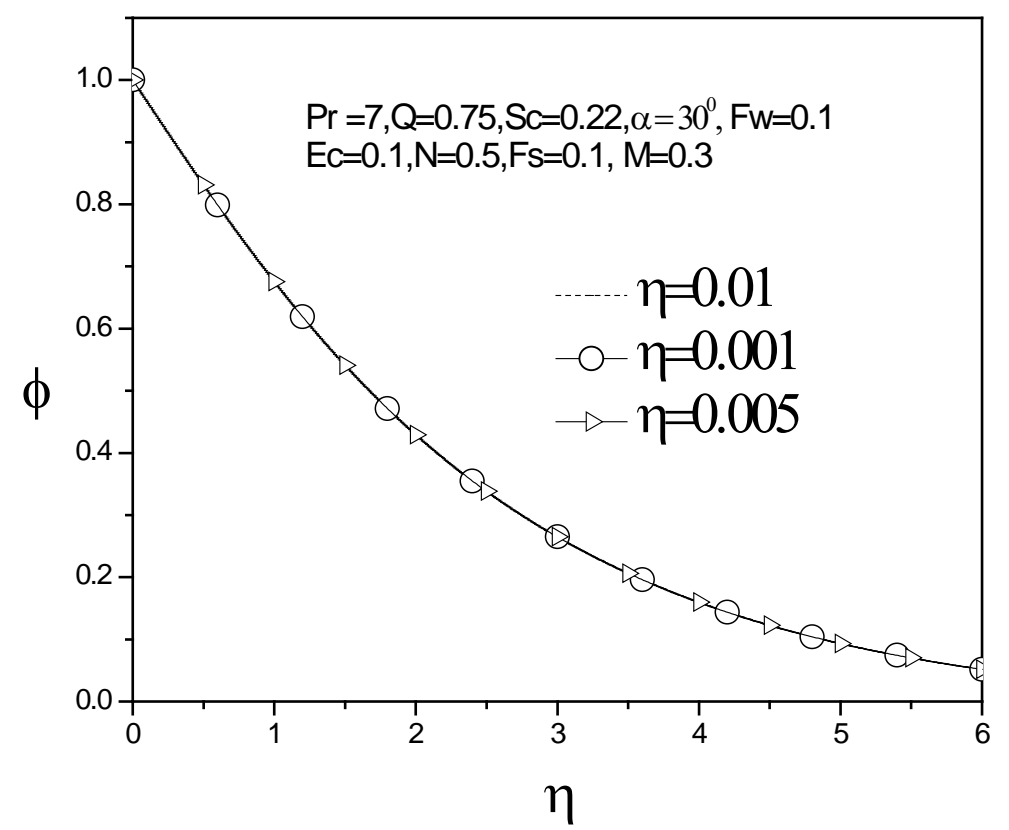

Figure 4. Concentration profiles for different step sizes.

ters are chosen arbitrarily where $\operatorname{Pr}=0.71$ corresponds physically to air at $20^{\circ} \mathrm{C} . \operatorname{Pr}=1$ corresponds to electrolyte solution such as salt water and $\operatorname{Pr}=7$ corresponds to water, and $S c=0.22,0.6$ and 1.0 correspond to hydrogen, water vapor and methanol respectively at approximate $25^{\circ} \mathrm{C}$ and 1 atmosphere.

Due to free convection problem positive large values of $\gamma=12$ is chosen. The value of $R e$ is kept 100 and $D a$ equal to 0.20 . However, numerical computations have been carried out for different values of the suction parameter $\left(F_{W}\right)$, magnetic field parameter $(M)$, angle of inclination $(\alpha)$, Prandtl number $(P r)$, heat source parameter $(Q)$, radiation parameter $(N)$ and Eckert number $E c$. The nu- 
merical results for the velocity, temperature and concentration profiles are displayed in Figures 5-25.

The effect of the angle of inclination $\alpha$ of the sheet on the velocity field is shown in the Figure 5. From this figure, we see that the velocity decreases with the increase of $\alpha$ swiftly up to $\eta=1.5$. After $\eta=1.5$, the velocity increases because the buoyancy force decreases. Figure 6 shows that temperatures rise with the grow of $\alpha$. Finally, we observe that the angle of inclination affects the concentration very slowly near the plate surface. Away from the plate, however, the effect on the concentration profile is significant.

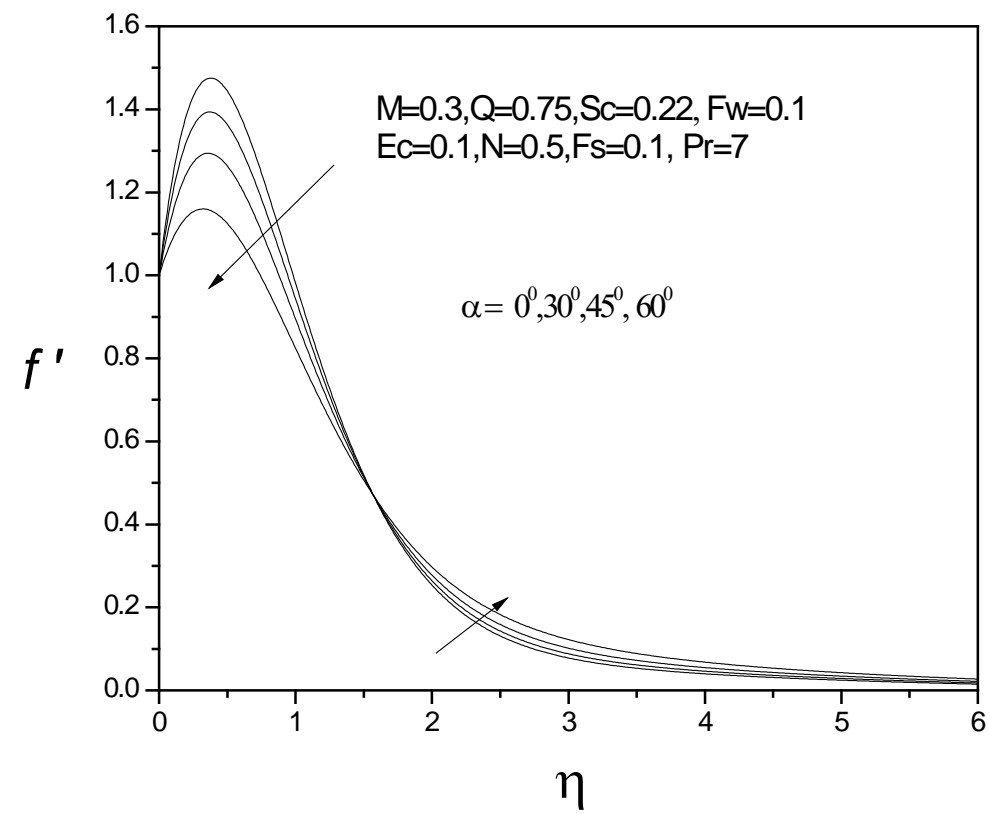

Figure 5. Velocity profiles for different values of $\alpha$.

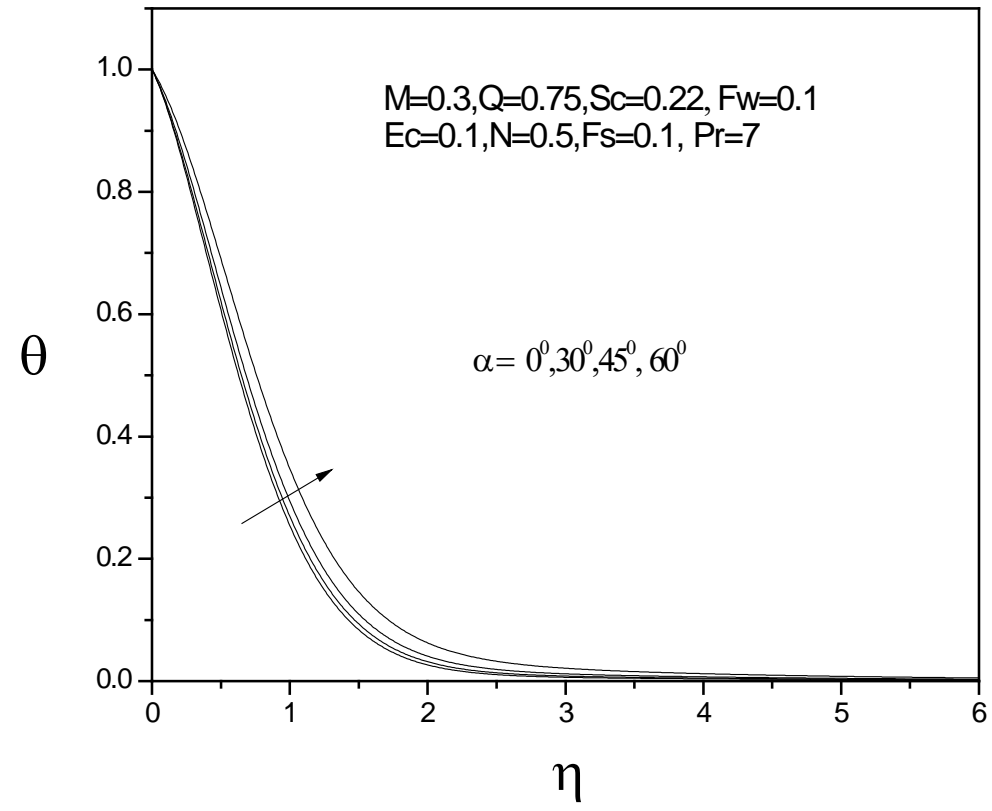

Figure 6. Temperature profiles for different values of $\alpha$. 


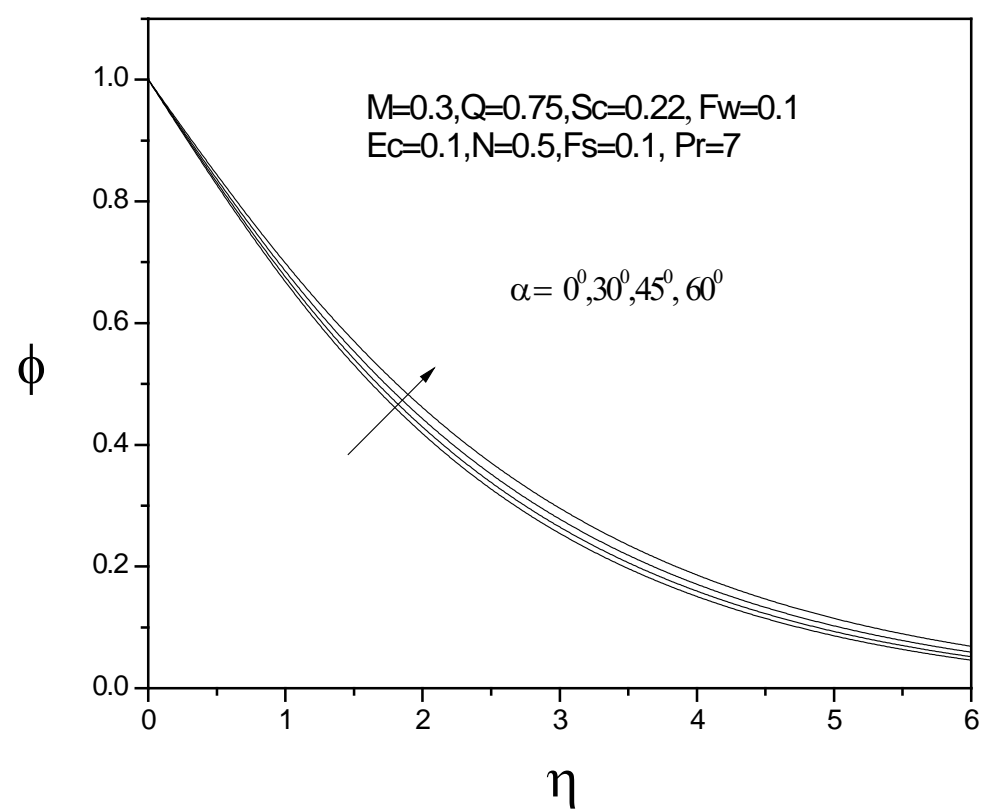

Figure 7. Concentration profiles for different values of $\alpha$.

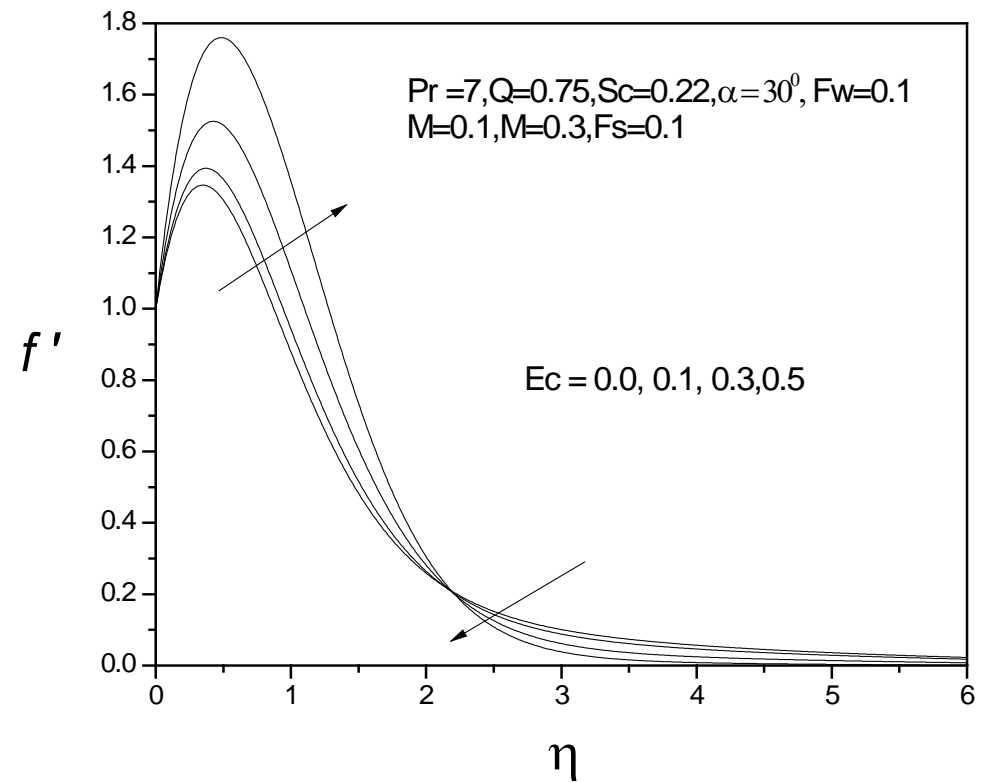

Figure 8. Velocity profiles for different values of $E c$.

Figures 8-10 are drawn to discuss the influence of Eckert number $E c$ on velocity, temperature and concentration profiles. Figure 8 shows that the velocity profiles increase with the increase of $E c$ upto $\eta=2.25$. After $\eta=2.25$ the velocity profiles reduce. Again Figure 9 shows quick increasing effect on temperature profiles. On the other hand, Ec has significant decreasing effect on concentration profiles observed in Figure 10.

Figure 11 to Figure 13 demonstrate the impact of the suction parameter $F_{W}$ on the velocity, temperature and concentration profiles. It is observed that when $F_{W}$ increases, the velocity, temperature and concentration decrease monotoni- 


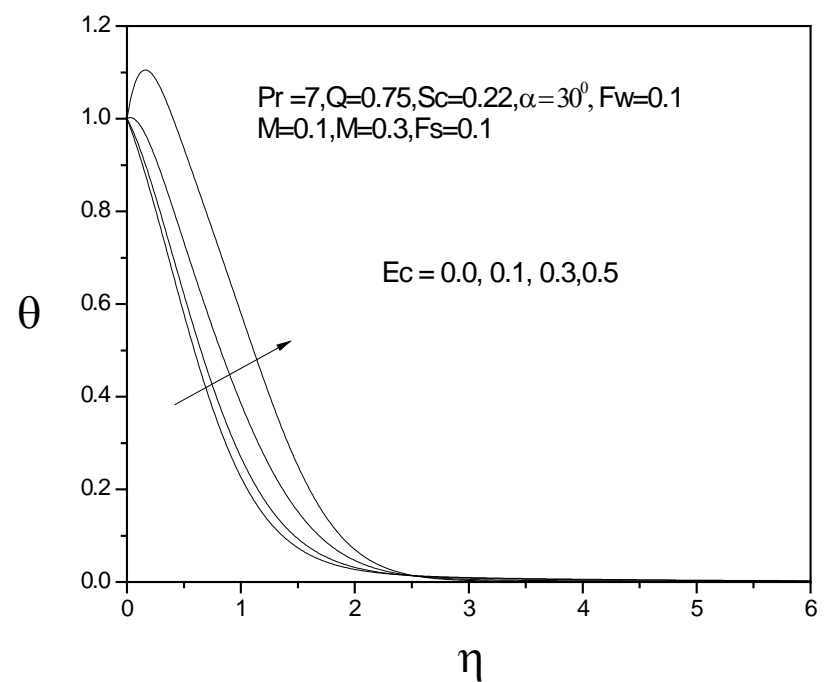

Figure 9. Temperature profiles for different values of $E \mathcal{c}$.

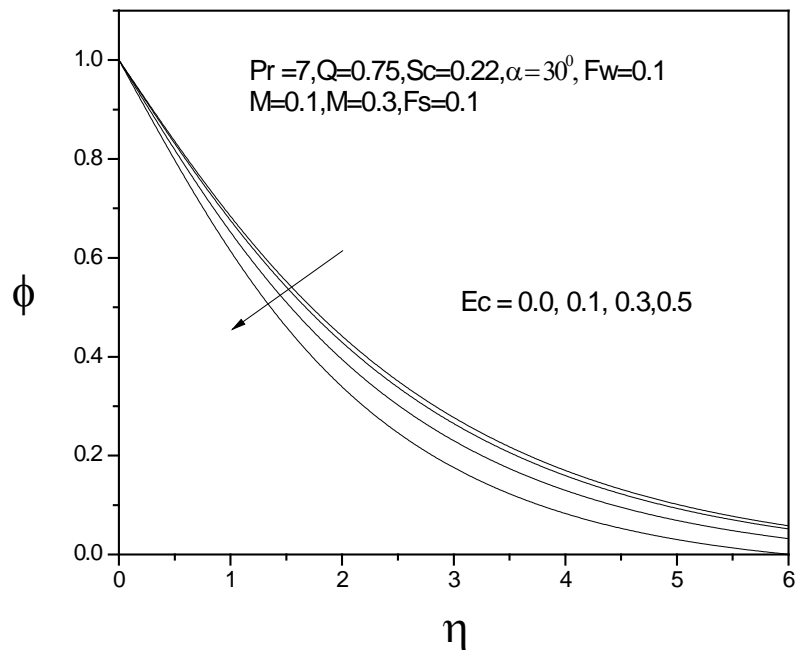

Figure 10. Concentration profiles for different values of $E c$.

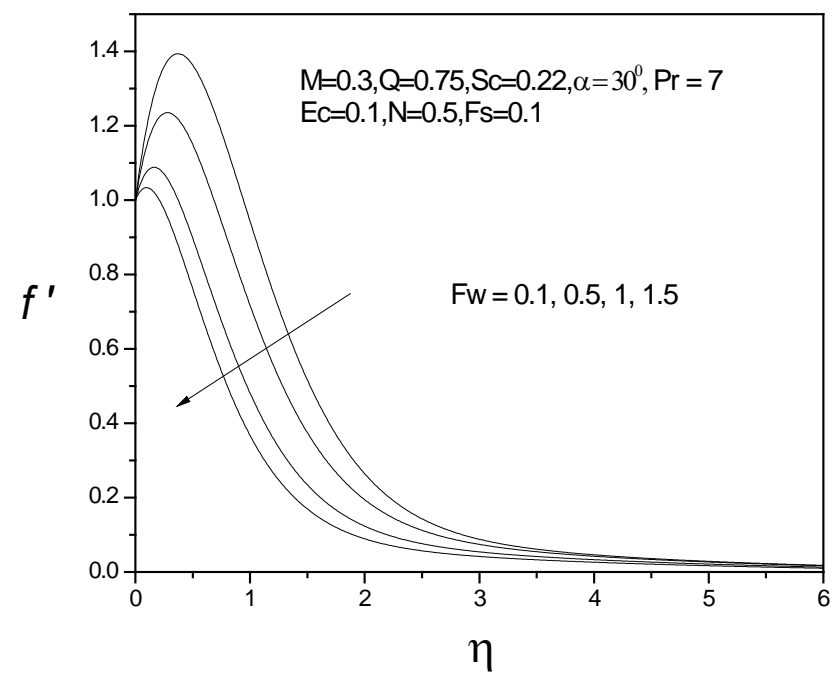

Figure 11. Velocity profiles for different values of $F w$. 


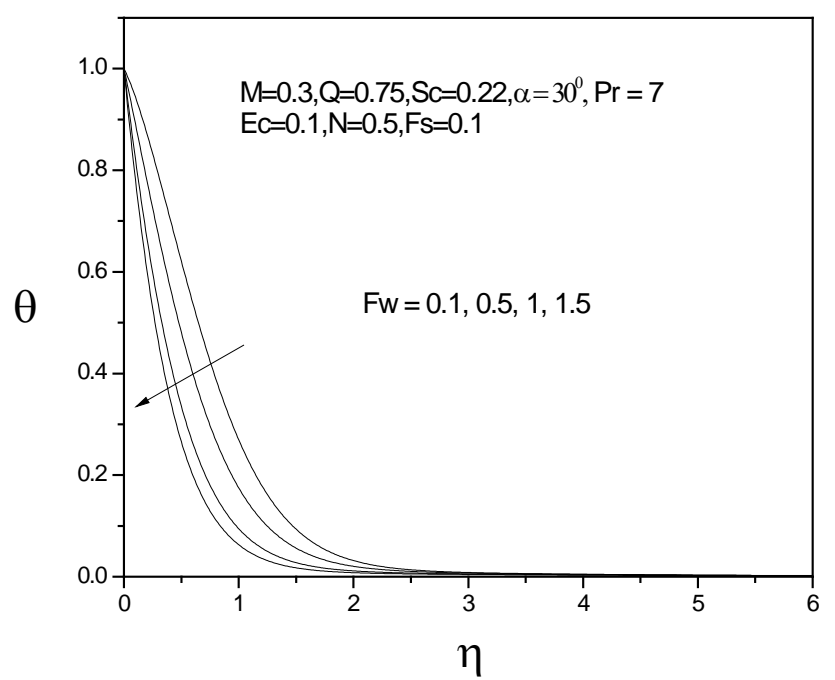

Figure 12. Temperature profiles for different values of $F_{W}$.

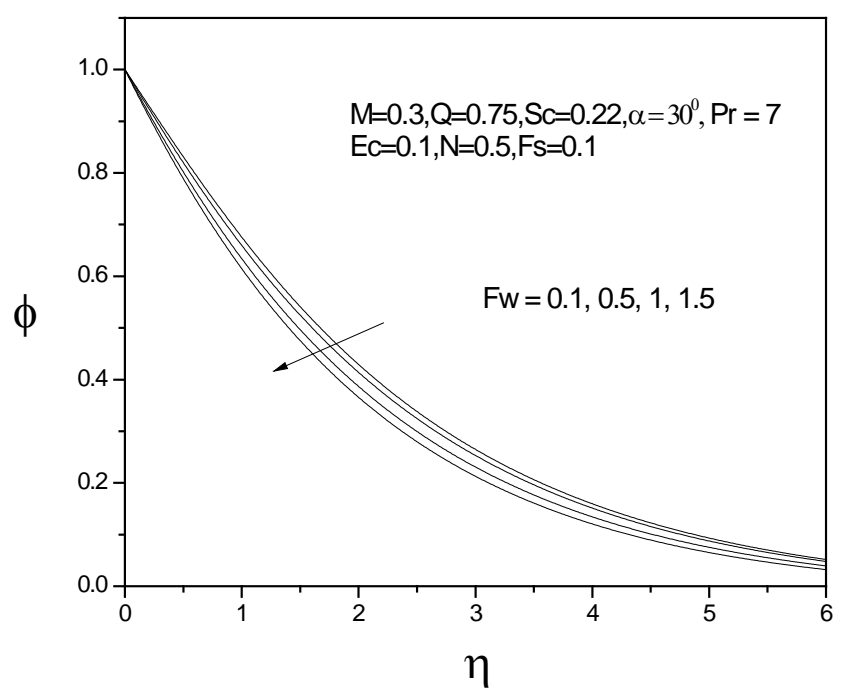

Figure 13. Concentration profiles for different values of $F W$.

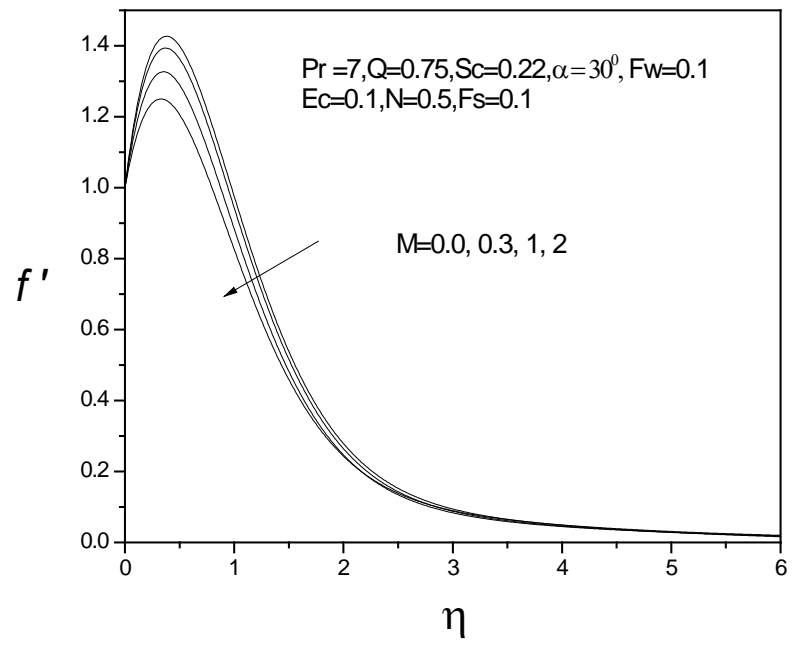

Figure 14. Velocity profiles for different values of $M$. 


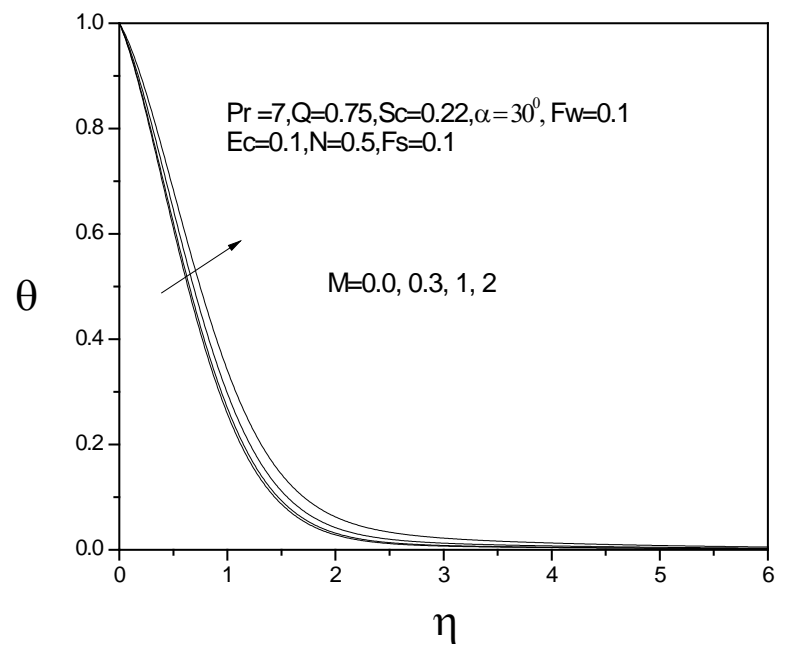

Figure 15. Temperature profiles for different values of $M$.

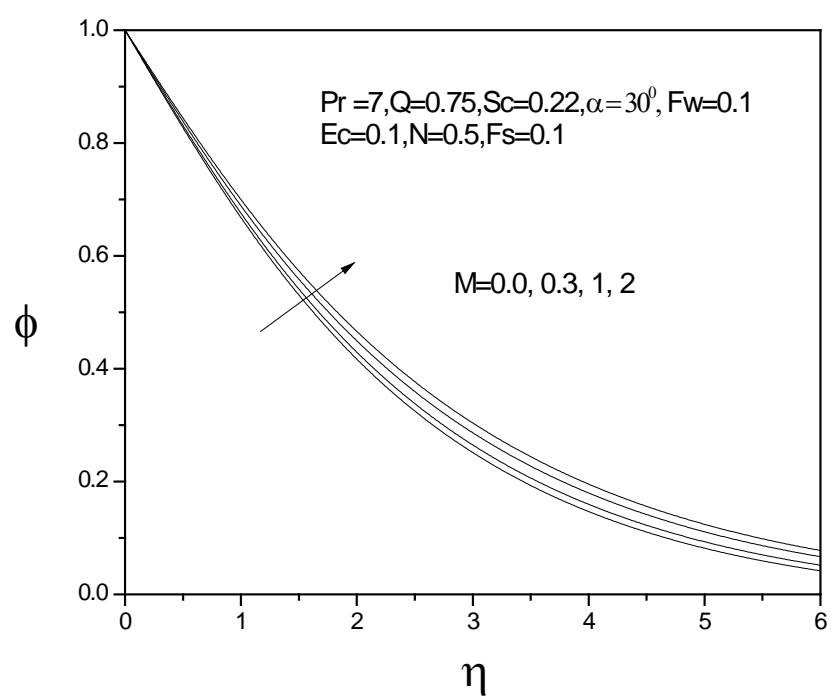

Figure 16. Concentration profiles for different values of $M$.

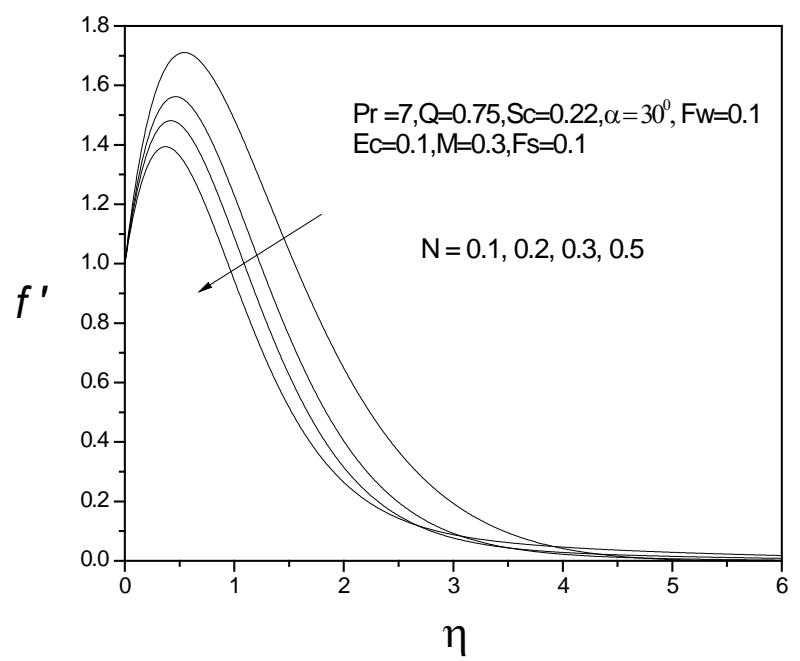

Figure 17. Velocity profiles for different values of $N$. 


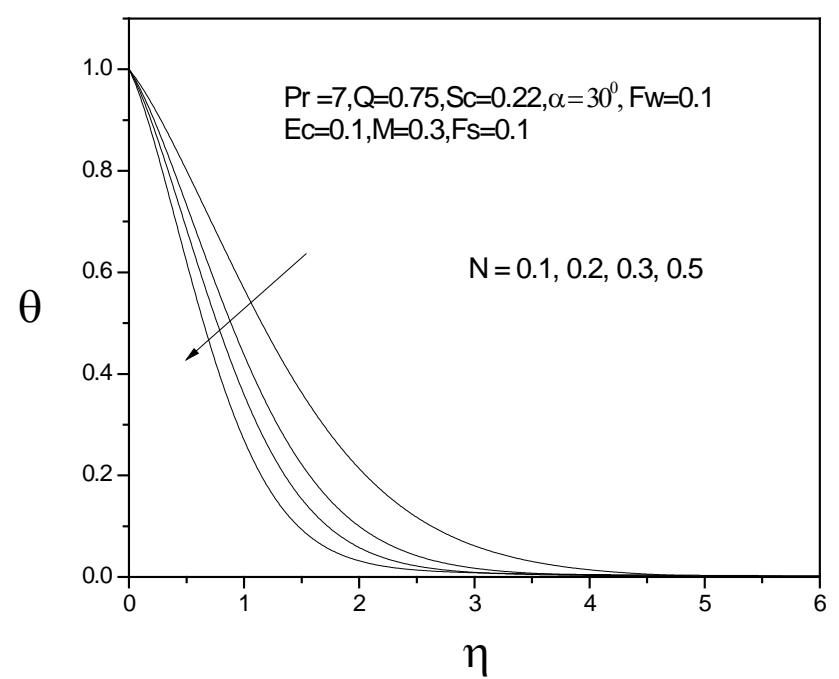

Figure 18. Temperature profiles for different values of $N$.

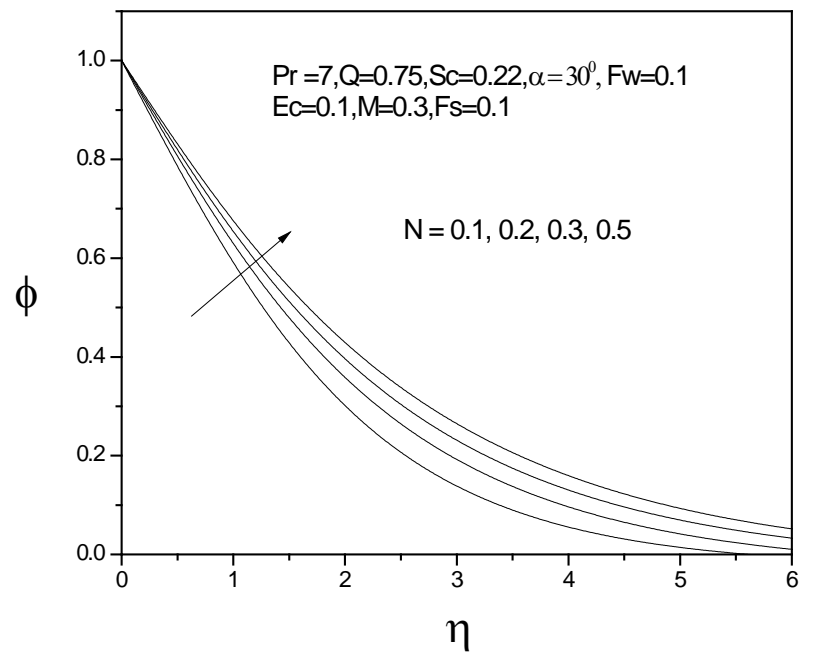

Figure 19. Concentration profiles for different values of $N$.

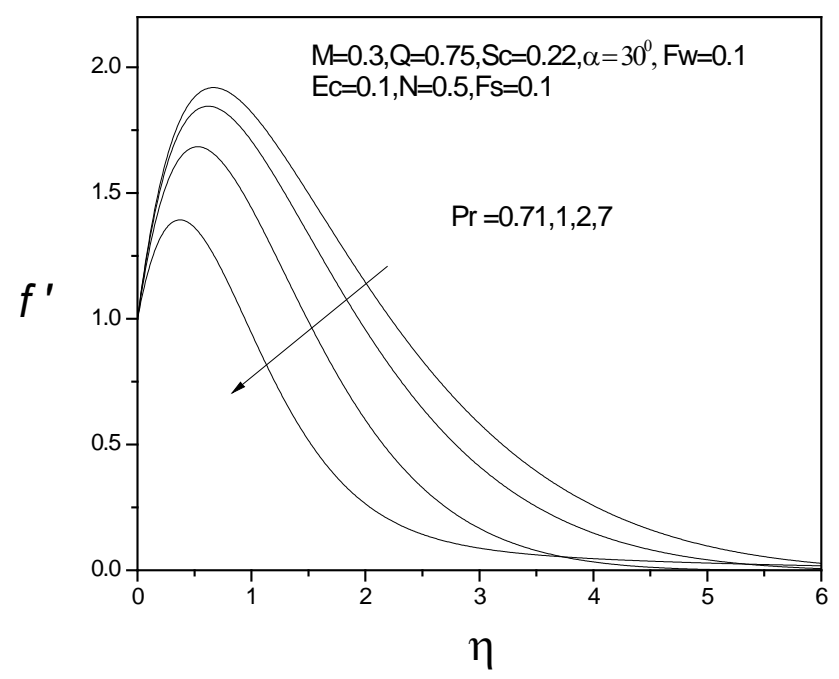

Figure 20. Velocity profiles for different values of Pr. 


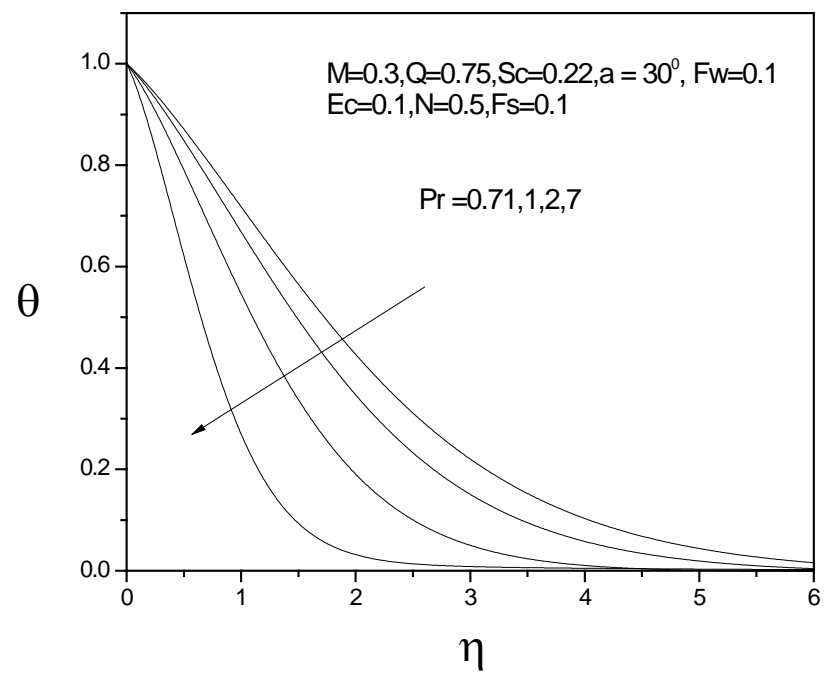

Figure 21. Temperature profiles for different values of $P r$.

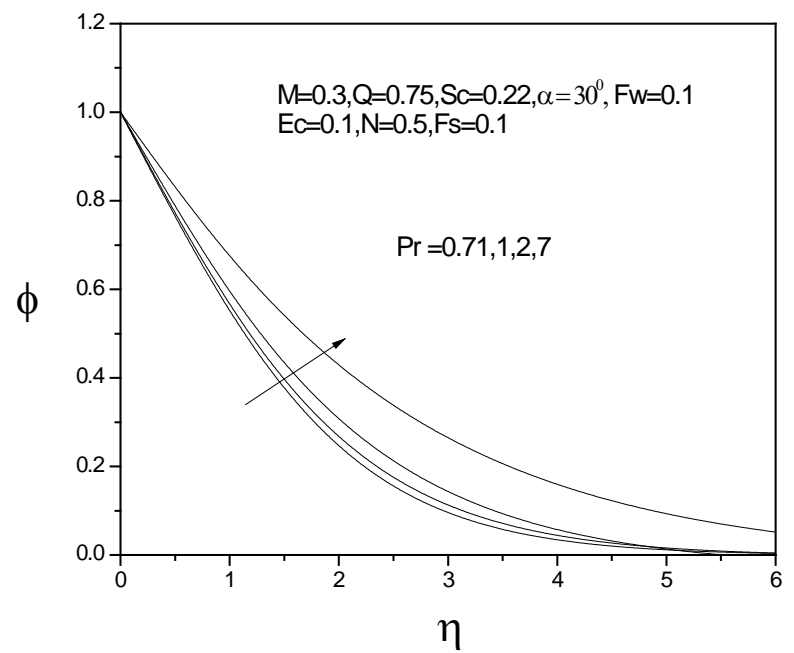

Figure 22. Concentration profiles for different values of $P r$.

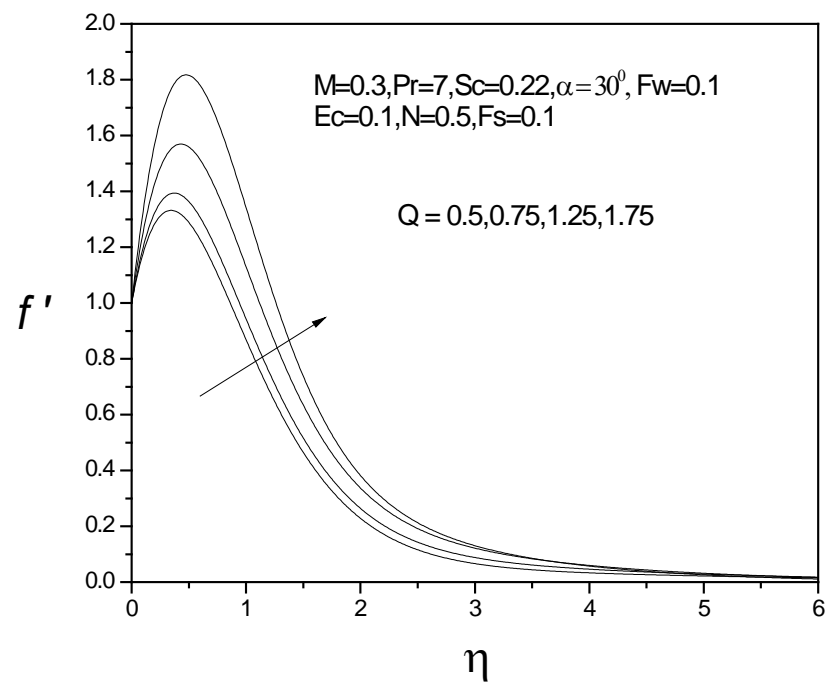

Figure 23. Velocity profiles for different values of $Q$. 


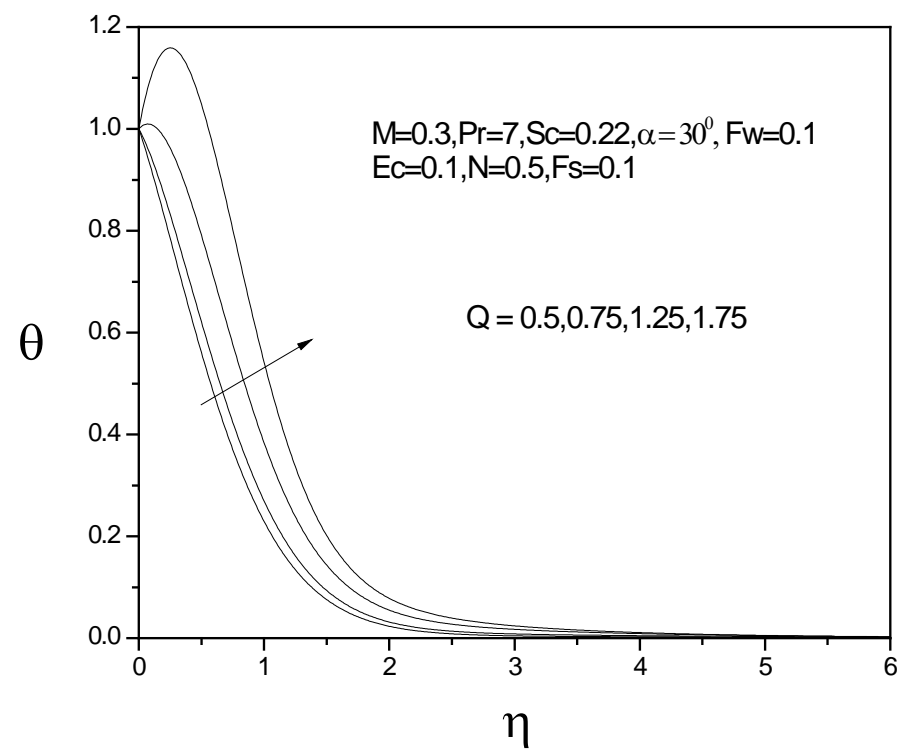

Figure 24. Temperature profiles for different values of $Q$.

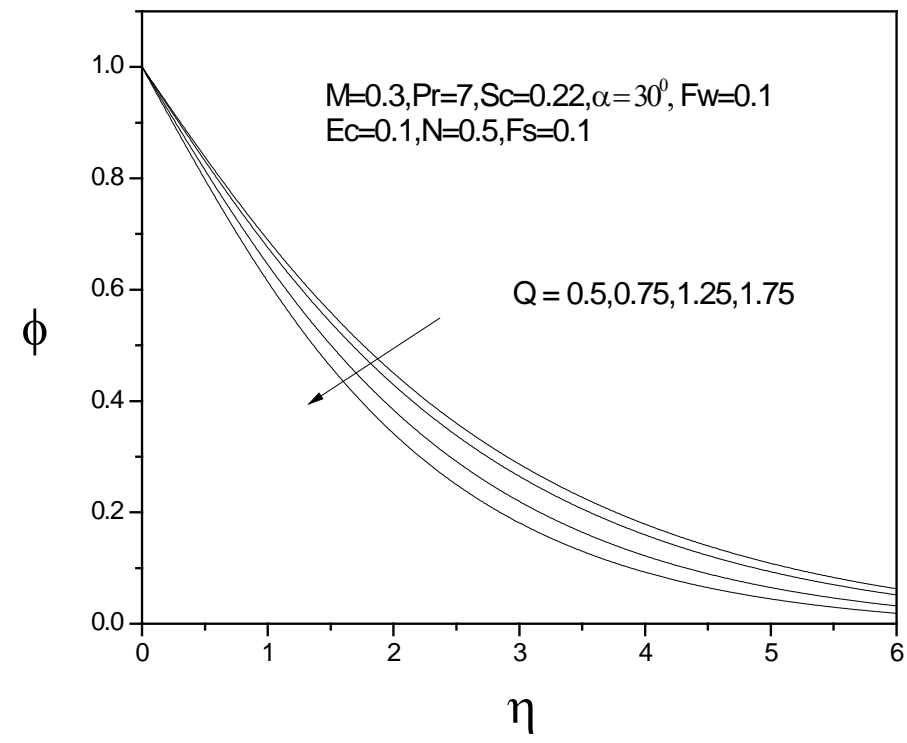

Figure 25. Concentration profiles for different values of $Q$.

cally. These figures indicate that since the boundary layer thickness getting smaller but the temperature and concentration gradient at the stretching sheet getting steeper, the increasing suction enhance the heat and mass transfer coefficient.

The influence of magnetic field parameter $M$ on velocity, temperature and concentration profiles are plotted in Figures 14-16. Here from Figure 14, we see that first the velocity decreases gradually up to $\eta=2.50$ as $M$ increases. On the other hand, the temperature as well as concentration profiles increase with the increase of $M$.

Figures 17-19 describe the dimensionless velocity, temperature and concentration profiles for different values of radiation parameter $N$. A strong decline in 
the velocity and temperature profiles are caused by increasing $N$ have found in Figure 17 and Figure 18. Again the concentration profiles are significantly increased for the increasing value of $N$ shown in Figure 19.

The behavior of Prandtl number Pr on velocity, temperature and concentration distributions are displayed in Figures 20-22. From the Figure 20 and Figure 21 , we see that the velocity profiles and the temperature profiles decrease rapidly with the increase of Prandtl number Pr. Again Figure 22 shows that the concentration profiles increase sharply as Prandtl number increases.

For different values of heat source parameter $Q$, the velocity, temperature and concentration profiles are illustrated in Figures 23-25. Here we have plotted non-dimensional velocity, temperature and concentration profiles against $\eta$ for some representative values of the heat source parameter

$Q=0.5,0.75,1.25,1.75$. The positive value of $Q$ represents source i.e., heat generation in the fluid. For heat generation, the peak velocity occurs near the surface of the stretching plate. This is corroborated by Figure 24 where it is seen that the temperatures do indeed rapid increase as $Q$ increases. Figure 25 shows that the concentration profiles decrease with the increase of heat source parameter.

\section{Conclusions}

The main goal of this study was the mathematical and numerical study of the viscous dissipation and radiation effect on MHD free convection flow past an inclined stretching sheet. The numerical solutions of the governing differential equations were obtained by using the shooting method. We observed the behavior of the physical parameters $\alpha, M, Q, N, E c, F w, \operatorname{Pr}$ and also commented the numerical results from their plots.

Finally, the effects of various parameters on the skin friction $C_{f}$, local Nusselt number $N u_{x}$ and local Sherwood number $S h$ for $\alpha=30^{\circ}, F w=0.1, M=0.3, \quad S C=0.22, F S=0.1$ are shown in the Table 1.

Table 1. $C_{f}, N u_{x}$ and $S h$ for different values

\begin{tabular}{ccccccc}
\hline$P r$ & $Q$ & $E c$ & $N$ & $C_{f}$ & $N u_{x}$ & $S h$ \\
\hline 0.71 & 0.5 & 0.1 & 0.5 & 3.40388 & 0.26724 & 0.46977 \\
0.71 & 0.75 & 0.1 & 0.5 & 3.5041 & 0.19956 & 0.47801 \\
0.71 & 1.25 & 0.1 & 0.5 & 3.74353 & 0.03763 & 0.49644 \\
7.0 & 0.75 & 0 & 0.5 & 2.25432 & 0.65081 & 0.33366 \\
7.0 & 0.75 & 0.1 & 0.5 & 2.40807 & 0.45194 & 0.34297 \\
7.0 & 0.75 & 0.3 & 0.5 & 2.81869 & -0.1642 & 0.36898 \\
7.0 & 0.75 & 0.1 & 0.1 & 3.14799 & 0.27511 & 0.43487 \\
7.0 & 0.75 & 0.1 & 0.2 & 2.83916 & 034083 & 0.39314 \\
7.0 & 0.75 & 0.1 & 0.3 & 2.64517 & 0.38711 & 0.36661 \\
\hline
\end{tabular}


We can make the following conclusions from the present study:

1. The effect of heat generation is remarkable. An increase in heat generation results in increasing velocity and temperature within the boundary layer.

2. Eckert number has effects on velocity as well as temperature profiles.

Radiation has significant effects on temperature profiles. So we can control the temperature field by using this parameter.

\section{References}

[1] Ostrach, S. (1952) Laminar Natural Convection Flow and Heat Transfer of Fluid with and without Heat Source in Channels with Constant Wall Temperature. NACA TN 2863.

[2] Hossain, M.A. and Takhar, H.S. (1996) Radiation Effects on Mixed Convection Along a Vertical Plate with Uniform Surface Temperature. Heat and Mass Transfer, 31, 243-248. https://doi.org/10.1007/BF02328616

[3] Ali, M.M., Chen, T.S. and B.F. Armaly (1984) Natural Convection Radiation Interaction in Boundary Layer Flow over Horizontal Surface. AIAA Journal, 22, 1797 1803. https://doi.org/10.2514/3.8854

[4] Mamsour, M.A. (1990) Radiation and Free Convection Effects on the Oscillatory Flow Past a Vertical Plate. Astrophysics and Space Science, 166, 269-275. https://doi.org/10.1007/BF01094898

[5] Alabrara, M.A., Bestman, A.R. and Ogulu, A. (1992) Laminar Convection in Binary Mixed of Hydromagnetic Flow with Radiation Heat Transfer. Astrophysics and Space Science, 195, 431-439. https://doi.org/10.1007/BF00646774

[6] Sattar, M.A. and Kalim, H. (1996) Unsteady Free Convection Interaction with Thermal Radiation in a Boundary Layer Flow past a Vertical Porous Plate. Journal of Mathematical and Physical Sciences, 30, 25-37.

[7] Chen, C.-H. (2004) Heat and Mass Transfer in MHD Flow by Natural Convection from a Permeable, Inclined Surface with Variable Wall Temperature and Concentration. Acta Mechanica, 172, 219-235. https://doi.org/10.1007/s00707-004-0155-5

[8] Anajali Devi, S.P. and Ganga, B. (2009) Viscous Dissipation Effects on Non-Linear MHD Flow in a Porous Medium over a Stretching Surface. International Journal of Applied Mathematics and Mechanics, 5, 45-59.

[9] Jha, B.K. and Ajibade, A.O. (2011) Effect on Viscous Dissipation on Natural Convection Flow between Vertical Parallel Plates with Time-Periodic Boundary Conditions. Thermophysics and Aeromechanics, 18, 561-571. https://doi.org/10.1134/S0869864311040056

[10] Ferdows, M., Nazmul and Ota, M. (2011) Thermophoresis and Chemical Reaction Effects on MHD Natural Convective Heat and Mass Transfer Flow in a Rotating Fluid Considering Heat and Mass Fluxes. Canadian Journal on Science and Engineering Mathematics, 2, 114-139.

[11] Hemly, K.A. (1995) MHD Boundary Layer Equations for Power Law Fluids with Variable Electric Conductivity. Meccanica, 30, 187-200.

https://doi.org/10.1007/BF00990456

[12] Nachtsheim, P.R. and Swigert, P. (1965) Satisfaction of the Asymptotic Boundary Conditions in Numerical Solution of the System of Non-Linear Equations of Boundary Layer Type. NASA TND-3004. 
Submit or recommend next manuscript to SCIRP and we will provide best service for you:

Accepting pre-submission inquiries through Email, Facebook, LinkedIn, Twitter, etc. A wide selection of journals (inclusive of 9 subjects, more than 200 journals)

Providing 24-hour high-quality service

User-friendly online submission system

Fair and swift peer-review system

Efficient typesetting and proofreading procedure

Display of the result of downloads and visits, as well as the number of cited articles Maximum dissemination of your research work

Submit your manuscript at: http://papersubmission.scirp.org/

Or contact ojfd@scirp.org 